\title{
Serum Anti-M ü llerian Hormone Levels in Multiple Sclerosis: A Case Control Study
}

Masoud mehrpour ${ }^{1}$, Fatemeh Taherian ${ }^{2 *}$, Paria Arfa-Fatollahkhani ${ }^{2}$, Abdorreza Naser Moghadasi ${ }^{3}$, Hosein Keivani ${ }^{4}$, Maryam Bahadori $^{2}$, Sara Mokhtar

${ }^{1}$ Department of Neurology, Firoozgar Hospital, Iran University of Medical Sciences, Tehran, Iran

${ }^{2}$ Department of Neurology, Rasoul-e-Akram Hospital, Iran University of Medical Sciences, Tehran, Iran

${ }^{3}$ MS Research Center, Neuroscience Institute, Tehran University of Medical Sciences, Tehran, Iran

${ }^{4}$ Department of Virology, Iran University of Medical Sciences, Tehran, Iran

${ }^{5}$ Department of Infertility, Avicenna Infertility Center, Tehran, Iran

*Corresponding author: Fatemeh Taherian, Department of Neurology, Rasoul-e-Akram Hospital, Iran University of Medical Sciences, Tehran, Iran, Tel: +989122039145 Fax: 982164352301; Email:f_m_taherian@yahoo.com

Received date: October 30, 2017; Accepted date: January 23, 2018; Published date: January 28, 2018

\section{Retraction Note:}

The article entitled "Serum Anti-Müllerian Hormone Levels in Multiple Sclerosis: A Case-Control Study," has been accepted for publication in the Journal of Neurology \& Neurophysiology considering the statements provided in the article as personal opinion of the author which was found not having any conflict or biasness towards anything. As the article was a perspective one, information provided by the author was considered as an opinion to be expressed through publication.

Publisher took decision to make the article online solely based on the reviewers suggestion which considered the article not but a personal opinion of the author. However, it is found that the author have some personal concerns and issues, therefore, being retracted from the journal. 